Hans-Gerd Happel

\title{
Urheberrecht in der Wissenschaft. Bericht über die 2. Urheberrechtskonferenz an der Europa-Universität Viadrina in Frankfurt (Oder) am 11.12.2019
}

https://doi.org/10.1515/bd-2020-0033

Der Lehrstuhl für Bürgerliches Recht und Privates Medienrecht unter der Leitung von Prof. Katharina de la Durantaye hat zum Ende des Jahres 2019 zusammen mit der Transferstelle und der Universitätsbibliothek im aufwendig restaurierten Logensaal der Universität, in einem der ältesten Gebäude in Frankfurt (Oder), eine Fachtagung zum Thema „Urheberrecht in der Wissenschaft“" organisiert. Damit konnte die $2018^{2}$ begonnene Tradition urheberrechtlicher Veranstaltungen im Rahmen des FDMentor-Projektes ${ }^{3}$ an der Viadrina erfolgreich fortgesetzt werden.

Nach der Begrüßung der ca. 70 Teilnehmenden aus zahlreichen Bundesländern stellte de la Durantaye die vortragenden Expertinnen und Experten vor und führte fachkundig in das Thema der Tagung ein, indem sie die Entwicklung des Urheberrechts in den letzten zwei Jahren anhand des UrhWissG ${ }^{4}$ vom 06.03.2018 und der DSM-Richtlinie ${ }^{5}$ vom 06.06.2019 kurz skizzierte ${ }^{6}$. Dabei betonte sie die insgesamt wissenschaftsfreundliche Entwicklung des Rechtsrahmens mit detail-

1 Eine Veröffentlichung der Vorträge über das Medienportal des IKMZ der Europa-Universität wird gegenwärtig vorbereitet, siehe https://mportal.europa-uni.de [Zugriff: 27.12.2019].

2 Hartmann, Thomas; Kaden, Ben; Kleineberg, Michael: Bericht zum Workshop „Rechtliche Aspekte bei digitalen Forschungsdaten“ an der Europa-Universität Viadrina Frankfurt (Oder). In: O-Bib. 5 (2018), Heft 2, S. 193-201, https://doi.org/10.5282/o-bib/2018H2S193-201 [Zugriff: 27.12.2019].

3 Helbig, Kerstin; Biernacka, Katarzyna; Buchholz, Petra; Dolzycka, Dominika; Hartmann, Niklas; Hartmann, Thomas; Hiemenz, Bea; Jacob, Boris; Kuberek, Monika; Weiß, Nadin; Dreyer, Malte: Lösungen und Leitfäden für das institutionelle Forschungsdatenmanagement. In: O-Bib. 6 (2019), Heft 3, S. 21-39, https://doi.org/10.5282/o-bib/2019H3S21-39 [Zugriff: 27.12.2019].

4 https://www.bundesgerichtshof.de/SharedDocs/Downloads/DE/Bibliothek/Gesetzesmateria lien/18_wp/UrhWissG/bgbl.pdf;jsessionid=AC98BC8697DEC95778F867B04ED4B83E.2_cid368?_ blob=publicationFile\&v=1 [Zugriff: 27.12.2019].

5 https://eur-lex.europa.eu/legal-content/DE/TXT/PDF/?uri=CELEX:32019L0790 [Zugriff: 27.12. 2019].

6 Eine Zusammenfassung der Kernpunkte des Gesetzes wird in einem kurzen Video von de la Durantaye leicht verständlich präsentiert: https://www.bildung-forschung.digital/de/mediavideo-8249.php [Zugriff: 27.12.2019]. 
liert definierten Nutzungsfreiheiten zu Gunsten von Bildung und Wissenschaft sowie die Bedeutung der im August 2019 vom BMBF und vom Deutschen Bibliotheksverband herausgegebenen, hilfreichen und praxisorientierten Broschüre „Urheberrecht in der Wissenschaft. Ein Überblick für Forschung, Lehre und Bibliotheken"7.

Auf diese begehrte und schon vielfach nachgedruckte Broschüre konzentrierte sich Bettina Klingbeil (Bundesministerium für Bildung und Forschung) in ihrem Eröffnungsvortrag „Urheberrecht in der Wissenschaft - eine Handreichung für die Praxis.“ Als Herausforderung für Bildung und Forschung in der digitalen Welt forderte sie eine Weiterentwicklung des wissenschaftsfreundlichen Rechtsrahmens, die Stärkung der Urheberrechtskompetenz sowie mehr Open Access-Aktivitäten. Da das UrhWissG auf fünf Jahre befristet ist, kommt es nun darauf an, so Klingbeil, die Erlaubnistatbestände der Paragrafen 60a bis 60d in Unterricht und Lehre, in der wissenschaftlichen Forschung und für das Text- und Datamining zu nutzen, um dann mit Hilfe der DSM-Richtlinie das nationale Recht untermauern zu können, sodass die Entfristung der mit dem UrhWissG geschaffenen Regelungen als Endziel erreicht werden kann.

Armin Talke (Staatsbibliothek zu Berlin) sensibilisierte in seinem Vortrag „Bildrechte - Fallstricke für Forschung und Lehre“ das Publikum anhand von praktischen Beispielen, wie komplex der Umgang mit der Präsentation und Publikation von Bildern ist. Erst nach Ablauf der urheberrechtlichen Schutzfrist von 70 Jahren nach dem Tod des Bildautors darf über ein Bild frei verfügt werden. Ansonsten muss jeder einzelne Vorgang betrachtet und eine Genehmigung für eine Publikation aufwendig eingeholt werden. Es gibt aber auch Ausnahmen (Beiwerk, Panoramafreiheit, Äußere Ansicht von öffentlichen Bauwerken), die problemlos fotografiert und veröffentlicht werden können. Erfreulich ist auch, dass umfangreiche Sonderregelungen für Unterricht und Lehre im UrhWissG definiert wurden, wenn sichergestellt ist, dass Bilder, Fotos und Grafiken usw. nur einem abgeschlossenen Kreis (z. B. mit Passwortschutz) zugänglich gemacht werden.

Für Forschungsorganisationen und Einrichtungen des Kulturerbes sieht Prof. Eric Steinhauer (Universitätsbibliothek der FernUniversität in Hagen) in seinem Vortrag „Jenseits von Plattformhaftung und Presseleistungsschutz. Was bringt die Urheberrechtslinie für Bildung, Wissenschaft und Bibliotheken?" eine positive Perspektive. Er kommt zu dem Schluss, dass die Befristung der Schranken für Lernplattformen, Text- und Datamining sowie Kulturerbeschutz als europa-

7 Bundesministerium für Bildung und Forschung: Urheberrecht in der Wissenschaft. Ein Überblick für Forschung, Lehre und Bibliotheken. Berlin 2019, https://www.bmbf.de/upload_ filestore/pub/Handreichung_UrhWissG.pdf [Zugriff: 27.12.2019]. 
rechtswidrig eingeschätzt werden kann. Die Evaluation der Schrankenregelungen durch den Gesetzgeber wird für die nicht-kommerzielle Nutzung nicht zu einer Verschlechterung der gegenwärtigen Situation führen, so die hoffnungsvolle und ermutigende Prognose Steinhauers. Sorgen bereiten Steinhauer dagegen die strengen Auflagen bei der Anfertigung von Digitalisaten vergriffener Werke. Hier hat der EuGH in einem Urteil $2016^{8}$ darauf hingewiesen, dass vergriffene Werke nur vervielfältigt werden dürfen, wenn der Urheber dazu persönlich Stellung genommen hat. Eine Information an den Urheber ohne Widerspruch reicht nach dieser Entscheidung nicht aus.

Innerhalb von nur 11 Monaten gelang es Prof. Ellen Euler (FH Potsdam) eine „Open-Access-Strategie für Brandenburg“9 mit Hilfe eines hochmotivierten Teams, welches sich aus Experten/innen der Universitäten und Hochschulen in Brandenburg zusammensetzte, fertigzustellen. In dem Strategiepapier werden Maßnahmen und Ziele definiert, die dazu führen sollen, einen nachhaltigen Transformationsprozess und den damit verbundenen Kulturwandel des Publizierens einzuleiten. Im Zentrum des Entwicklungsprozesses wird die Implementierung einer Vernetzungs- und Kompetenzstelle (VUK OA) stehen, die professionell und zentral gesteuert an den einzelnen Hochschulen des Landes Brandenburg unterstützend und beratend eingreifen wird.

Aus dem Nachbarland Polen berichtete Prof. Marek Niezgodka (Kardinal-Stefan-Wyszynski-Universität Warschau) zum Schluss der Tagung über „Open Science in Poland: Past developments, current challenges, future perspectives“. Der mit führenden Wissenschaftseinrichtungen in Polen und Deutschland sowie zahlreichen weiteren europäischen Ländern eng vernetzte Wissenschaftler und Wissenschaftsmanager vom Center for Digital Science and Technology ist ein ausgewiesener Experte und erfolgreicher Verhandlungsführer für qualitätsvolle Open Access-Informationen und kommerzielle Lizenzen. Beeindruckend demonstrierte er, wie in Polen auf nationaler Ebene leistungsfähige und benutzerfreundliche Portale $^{10}$ aufgebaut werden, die unter Beachtung der FAIR-Principles ${ }^{11}$ weiterentwickelt werden und in das Konzept der European Science Cloud ${ }^{12}$ integriert werden sollen.

8 EuGH Pressemitteilung Nr. 126/16 vom 16.11.2016. Rechtssache C-301/15, https://curia.europa. eu/jcms/upload/docs/application/pdf/2016-11/cp160126de.pdf, https://eur-lex.europa.eu/legalcontent/DE/TXT/PDF/?uri=CELEX:32019L0790 [Zugriffe: 27.12.2019].

9 https://zenodo.org/record/2581783\#.XgYyxO-WyUk [Zugriff: 27.12.2019].

10 Z.B. Wirtualna Biblioteka Nauki: https://www.wbn.edu.pl und Center for Open Science: https://www.otwartanauka.pl [Zugriffe: 30.12.2019].

$11 \mathrm{https://www.go-fair.org/fair-principles} \mathrm{[Zugriff:} \mathrm{30.12.2019].}$

12 https://www.eosc-portal.eu [Zugriff: 30.12.2019]. 
Nach den Vorträgen wurden zahlreiche Fragen und anregende Diskussionsbeiträge geäußert, die in den Pausen an runden Tischen vor dem Logensaal lebhaft fortgesetzt wurden. Ein zentrale Fragestellung stand dabei immer wieder im Vordergrund: Wie schaffen wir es, dass an den Hochschulen und wissenschaftlichen Einrichtungen ein zentraler Beratungsservice für Open Access, Open Science und Forschungsdatenmanagement ${ }^{13}$ aufgebaut wird, der Urheberrechte und auch weitere damit im Zusammenhang stehende Rechtsgebiete berücksichtigt? Wer könnte diesen komplexen Aufgabenbereich übernehmen? Wird es hier zukünftig eine Ansprechpartnerin oder einen Ansprechpartner vor Ort in den einzelnen Einrichtungen geben? Die Übernahme welcher neuen Aufgaben werden hier dabei von den Bibliotheken ${ }^{14}$ erwartet?

Eindeutige Antworten und Patentrezepte gibt es nicht, da waren sich die Teilnehmenden einig. Die Transformation der Wissenschaft zu einem auf Open Access basierenden, wissenschaftlichen Publikationswesen geht unaufhaltsam weiter. Diesem Entwicklungsprozess folgend müssen dann auch leistungsfähige Strukturen und professionelle Beratungsangebote geschaffen werden. Vielleicht können auf der nächsten Urheberrechtstagung in Frankfurt (Oder) dazu schon konkrete Umsetzungen und neue Perspektiven präsentiert werden.

\author{
Hans-Gerd Happel \\ Direktor der Universitätsbibliothek Frankfurt (Oder) \\ Große Scharrnstraße 59 \\ 15230 Frankfurt (Oder) \\ E-Mail: happel@europa-uni.de
}

13 Vgl. dazu die Grafik von Thomas Hartmann, FDMentor-Mitarbeiter an der Europa-Universität Frankfurt (Oder) wie Anmerkung 3, S. 30.

14 Franke kommt nach der Durchführung einer Umfrage zu dem folgenden Ergebnis: „Da das Urheberrecht alle Bereiche der Bibliothek betrifft, müssen alle Bibliothekar/innen Kenntnisse über das Urheberrecht besitzen“. Franke, Fabian: Urheberrechtskompetenz - ein Thema für Bibliotheken?! In: O-Bib. 6 (2019), Heft 4, S. 151-163, hier S. 163, https://doi.org/10.5282/obib/2019H4S151-163 [Zugriff: 30.12.2019]. 